\title{
Investigación Básica
}

\section{Angiotensina-(1-9) disminuye el remodelamiento cardiovascular hipertensivo independiente de los niveles de ECA y de angiotensina II.}

Jackeline Moya ${ }^{1}$, Ulises Novoa ${ }^{1}$, Iván Godoy ${ }^{1}$, Mario Chiong ${ }^{2}$, Sergio Lavandero², Jorge Jalil1, María Paz Ocaranza ${ }^{1}$.

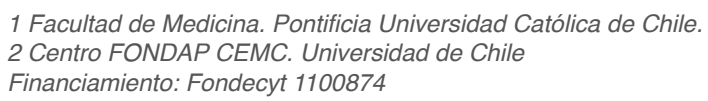

\section{Resúmen}

La enzima convertidora de angiotensina I (ECA2) a través de Angiotensina (Ang) $\neg$ (1-9) más que Ang-(1-7) contrarresta los efectos deletéreos de ECA y Ang II. Se desconoce si Ang-(1-9) es efectiva en el tratamiento del remodelamiento cardiovascular (RMCV) hipertensivo, en ratas con polimorfismo del gen de la ECA.

Objetivo: Determinar el efecto de Ang-(1-9) en el tratamiento del RMCV hipertensivo en ratas con niveles genéticamente determinados de ECA y Ang II.

Métodos: Ratas normotensas homocigotas, Lewis (LL) y Brown Norway (BN), se les indujo HTA a través del modelo Goldblatt (GB, 2 riñones-1 pinzado). Después de 4 semanas, las ratas hipertensas se randomizaron para recibir Ang-(1-9) (602 ng/Kg min) o una coadministración de Ang-(1-9)+A779 (100 ng/Kg min, antagonista del receptor MAS de Ang-(1-7)) durante 14 días mediante una minibomba. Como controles se usaron ratas sometidas a operación ficticia (Sham). Se determi- nó masa corporal (MC), presión arterial sistólica (PAS), masa ventricular (MV), área de cardiomiocitos (AC), área y grosor de la túnica media (ATM, GTM), fracción volumétrica de colágeno total (FVCT) en el ventrículo izquierdo (VI), niveles proteicos de colágeno tipo I (Col I) en la aorta (Ao) y la infiltración de macrófagos en Ao y VI, por medio de su molécula especifica ED1 (ED1-Ao, ED1-VI).

Resultados: La administración de Ang-(1-9) disminuyó significativamente PAS, MV, AC, FVCT, Col I, ATM, GTM, ED1-Ao (-) y ED1 $\neg$ VI, en las ratas hipertensas LL y BN respecto a las ratas GB sin tratamiento, respectivamente. Este efecto no fue inhibido por el antagonista A779. El polimorfismo de la ECA no modificó la respuesta al tratamiento.

Conclusión: Ang-(1-9) redujo eficazmente la HTA y el RMCV secundario, independiente al polimorfismo en el gen de la ECA. Este efecto posiblemente es directo ya que no fue mediado por Ang-(1-7). Fondecyt 1100874 .

Correspondencia:

María Paz Ocaranza

Departamento de Enfermedades Cardiovasculares. Facultad de Medicina. P.

Universidad Católica de Chile.

Tel: +562-3543407, Fax: +562-6321924.

mocaran@med.puc.cl 


\section{Angiotensin-(1-9) reduces hypertensive cardiovascular remodeling independent of ACE and Ang II levels.}

Background: The angiotensin I converting enzyme 2 (ACE2) counteracts the deleterious effects of ACE and Ang II through angiotensin (Ang) -(1-9) rather than Ang-(1-7).In addition, it is not clear whether Ang-(1-9) is effective in the reversal of hypertensive cardiovascular remodeling (CVRM) in rats with ACE gene polymorphism.

Objective: To determine the effect of Ang-(1-9) in the prevention of hypertensive CVRM in rats with genetically determined levels of ACE and Ang II.

Methods: In normotensive homozygous Lewis (LL) and Brown Norway $(\mathrm{BN})$ rats hypertension was induced by the Goldblatt 2 kidney-1 pinch model. After 4 weeks, rats were randomized to receive Ang- (1-9) (602 ng / Kg min) or the co administration of Ang- (19) +A779 (100 ng / kg min, a MAS receptor antagonist of Ang- (1-7)) for 14 days. Sham operated rats were used as controls. We determined body mass (BM), systolic blood pressure (SBP), ventricular mass (VM), car- diomyocyte area (CA), area and thickness of the aortic media (ATM, TTM), LV total collagen volume fraction (FVCT), type I collagen protein levels (Col I) in the aorta (Ao) and macrophage infiltration in LV and Ao, through its specific molecule ED1 (ED1-Ao, ED1-VI). Results: Continuous administration of Ang- (1-9) significantly decreased SBP, VM, CA, TCVF, Col I, TTM, and ED1 in the aorta and left ventricle of hypertensive rats. This effect was not inhibited by the antagonist A779. ACE polymorphism did not modify the response to treatment.

Conclusion: Ang- (1-9) effectively reduced hypertension induced CVRM independent of ACE gene polymorphism. This effect was not mediated by Ang(1-7).

Fondecyt 1100874.

Keywords: Angiotensin I converting enzyme 2, ACE gene polymorphism, Ang- (1-9)

La sobreactivación del Sistema Renina Angiotensina (SRA) es uno de los principales factores fisiopatológicos asociados al desarrollo de la HTA, ${ }^{7}$ específicamente la vía clásica del SRA conformada por la enzima convertidora de angiotensina I (ECA) y su producto angiotensina (Ang) II. La Ang II al unirse a su receptor AT1 (RAT1) activa las vías de señalización que desencadenan un aumento de la presión arterial (PA) y cambios celulares, no celulares y funcionales en los distintos tejidos blanco como corazón y vasos, entre otros. ${ }^{4}$

La inhibición del SRA clásico a nivel de la ECA (IECA) y del RAT1 (ARA II) han sido las principales estrate- 
gias farmacológicas para el tratamiento de la HTA y del RMCV. Aunque ambos tipos de fármacos son eficientes para reducir la PA, no han mostrado igual eficacia en disminuir los efectos patológicos del RMCV hipertensivo y por consiguiente el daño en órganos blanco persiste. ${ }^{8}$

El descubrimiento de la ECA homóloga (ECA2) ${ }^{9,10}$ estableció una nueva vía paralela del SRA. En esta vía la $\mathrm{ECA}^{2}$ compite con la ECA por la hidrólisis de Ang I para formar Ang-1-9 ${ }^{10}$ además ECA ${ }^{2}$ transforma Ang II a Ang-1-7 ${ }^{11-12}$ Es conocido que Ang-1-7 presenta acciones opuestas a las de Ang II, al unirse a su receptor MAS, ${ }^{13}$ mediando la vasodilatación y efectos anti-hipertróficos, anti-inflamatorios y anti-fibróticos. ${ }^{13-14}$ En relación a la actividad biológica de Ang-1-9, los únicos antecedentes disponibles han sido recientemente publicados por nuestro grupo, y muestran que Ang-1-9, previene la hipertrofia cardiomiocitaria in vivo e in vitro. ${ }^{15}$ Además, hemos observado una disminución significativa de los niveles de Ang-1-9, y de ECA2 en la pared aórtica de animales con HTA y remodelamiento cardiovascular. ${ }^{16}$ El tratamiento con Ang-1-9, a ratas hipertensas por infusión de Ang II, previno la HTA y el remodelamiento cardiovascular secundario, de manera directa y fue independiente de la acción de Ang-1-7 ${ }^{17,18}$ Estos hallazgos junto con que Ang${ }^{1-9}$, es parte de un eje opuesto al SRA clásico apoyan el concepto que este péptido podría permitir el control de la PA y disminuir eficazmente el del RMCV secundario. Dado que hemos encontrado una compleja interrelación entre ECA y ECA2 y entre Ang II y Ang-1-9, ${ }^{15,19}$ el objetivo de este estudio fue evaluar el efecto de la administración de Ang-(1-9) en el RMCV hipertensivo en un modelo genético en rata que expresa distintos niveles circulantes de ECA y Ang II, y cómo se modifica por efecto de la HTA renovascular dependiente de renina. Este modelo de ratas homocigotas Lewis (LL) y Brown Norway (BN) ha sido ampliamente utilizado en nuestras investigaciones previas, ${ }^{20}$ describiendo que en condiciones basales, las ratas $\mathrm{BN}$ presentan mayores niveles de ECA, renina y Ang II pero baja actividad de NEP y Ang-1-7. ${ }^{20,21}$ En cambio, las ratas LL presentan características opuestas. Por otra parte, el pinzamiento de la arteria renal izquierda (modelo Goldblatt, GB, 2K-1C) genera mayor HTA en las ratas BN que las LL. ${ }^{20}$

\section{Materiales y Métodos}

El estudio se realizó según la "Guía para el cuidado y uso de animales de laboratorio" publicado por el "National Health Institute" (NIH No 85-23, 1985) y aprobada por la Comisión de Investigación de la Facultad de Medicina de la Pontificia Universidad Católica de Chile.
1. Modelo experimental: Se usaron ratas homocigotos (BN y LL) machos normotensas (peso $150 \pm 10 \mathrm{~g}$ ), de $2^{\circ}$ generación las cuales presentan un polimorfismo en el gen ECA. ${ }^{20}$ Las ratas fueron randomizadas a operación Goldblatt (GB; 2Riñons-1Clip). ${ }^{22}$ Como grupo control (S), se usaran ratas sometidas a similares condiciones de estrés. A la 4ta semana (sem) post-cirugía, las ratas con hipertensión demostrada (PAS $\geq 140 \mathrm{mmHg}$ ) fueron randomizadas a recibir una administración crónica de Ang-1-9 [ 602 ng kg-1min-1] o una mezcla de Ang-1-9 con A779, ${ }^{23}$ un antagonista del receptor MAS de Ang-(1-7) [ 100 ng kg1min-1], por un periodo de 14 días usando minibombas osmóticas implantadas en la vena yugular.

2. Parámetros hemodinámicos: La PAS fue medida semanalmente por pletismografía en la cola de las ratas previamente anestesiadas. ${ }^{24}$

3. Obtención del tejido ventricular: Al final del tratamiento, los animales fueron anestesiados con Ketamina $(35 \mathrm{mg} / \mathrm{kg})$ y Xilacina $(7 \mathrm{mg} / \mathrm{kg})$ por vía intra-peritoneal. El miocardio y la aorta fueron extraídos y lavados en suero fisiológico, una parte del tejido fue fijado en bouin e incluido en parafina. El resto del tejido fue conservado a $-80^{\circ} \mathrm{C}$.

4. Determinación de hipertrofia cardiovascular: $\mathrm{Se}$ utilizaron cortes transversales de aorta (Ao) y ventrículo izquierdo (VI) de $5 \mathrm{~mm}$ de espesor teñidos con hematoxilina-eosina. Las imágenes fueron tomadas mediante una cámara acoplada a un microscopio (Nikon) y cuantificadas utilizando un software (Nis-Element). En la Ao se registró el área del lumen (AL, mm2) y total (AT, mm2) del anillo aórtico (4X). El área de la túnica media (ATM, mm2), se obtuvo de la diferencia entre AT y AL.25 Para determinar el grosor de la túnica media (GTM, mm) se realizaron 10 mediciones por cada sección de anillo aórtico (40X). ${ }^{16}$ En el VI, se midió el área (AC, mm2) y perímetro de los cardiomiocitos (PC $\mu \mathrm{m}) .{ }^{15,26} \mathrm{Se}$ analizaron al menos 70 imágenes celulares por animal, las que se seleccionaron aleatoriamente. Además, el grado de hipertrofia cardíaca se evaluó de acuerdo a los parámetros de masa corporal (MC, gr) y masa ventricular (MV, gr).

\section{Evaluación de la fibrosis en el miocardio: Cortes} transversales de VI de $5 \mu \mathrm{m}$ de grosor fueron teñidos con rojo picrosirio. ${ }^{27}$ Las imágenes fueron captadas con una cámara digital acoplada a un microscopio. Con el aumento de $20 \mathrm{X}$ se tomaron fotos sucesivas hasta completar en- 
tre 60-80 fotos aproximadamente. Finalmente, se fijó el lente en una zona carente de tejido y se captó una foto blanco, con el propósito identificar los espacios sin tejido. Estas imágenes fueron analizadas con el programa Matlab diseñado para la medición de Colágeno, bajo protocolo establecido en el Laboratorio. ${ }^{20}$ Finalmente, se determinó el promedio de la fracción volumétrica de colágeno total (FVCT, \%).

6. Determinación de la fibrosis de la pared aórtica: Se utilizaron $50 \mathrm{mg}$ de tejido aórtico que fueron homogenizados en tampón de lisis y centrifugado a $10000 \mathrm{~g}$ por $30 \mathrm{~min}$ a $4^{\circ} \mathrm{C} .{ }^{28} \mathrm{El}$ contenido de proteínas se midió por el método de Bradford. ${ }^{29}$ Para el Western blot se utilizaron $50 \mu \mathrm{g}$ de proteína total de Ao, siguiendo el protocolo descrito por Rivera et al., 2007. Utilizando como anticuerpo primario, anti Col I (dilución 1:3000, AB749 Chemicon) y anticuerpo segundario, anti-IgG de conejo conjugado a peroxidasa (dilución 1:10000, Pierce 31460). Como control de carga se utilizó $\alpha$-actina, empleando un anticuerpo anti a-actina (Sigma A5316) con una dilución de 1:5000 y como anticuerpo secundario, anti-IgG de ratón conjugado a peroxidasa (Piarce 31457) en dilución 1: 10000.

7. Determinación de la inflamación cardiovascular: Cortes transversales de $4 \mu \mathrm{m}$ de grosor de Ao y VI fueron desparafinados para luego realizar desenmascaramiento antigénico del tejido con EDTA $1 \mathrm{mM}$ a pH $8 .{ }^{30} \mathrm{La}$ inmunomarcación se realizó con el Kit DAKO K0679. Los cortes se incubaron con anti-ED1 de macrófagos de rata (Serotec MCA341R) en una dilución 1/200 (Ao) y 1/400 (VI) toda noche en una cámara húmeda a $4^{\circ} \mathrm{C}$. Posteriormente, los tejidos se lavaron y se incubaron con el anticuerpo secundario biotinilado durante $30 \mathrm{~min} \mathrm{~T}^{\mathrm{o}}$ ambiente. Como método de detección se utilizó la técnica de Diaminobenzidina (Kit DAKO) y contra tinción con hematoxilina. El número de células marcadas con ED-1 se midieron de forma cuantitativa para la Ao, ${ }^{31}$ evaluando la razón entre el número total de células positivas para ED1 y el área de anillo aórtico (ED1/A, mm2). Para el miocardio se realizó una evaluación semi-cuantitativa estableciéndose una escala de células (Céls) positivas (+) para ED1. ${ }^{32}$

8. Análisis de resultados: Los datos obtenidos se expresaron como promedio \pm error estándar de la media (SEM). Cada grupo experimental estuvo constituido por 4-8 animales. Las comparaciones entre los grupos se realizaron mediante Kruskal-Wallis, seguido de un análisis de Mann-Whitney. Para el análisis semi-cuantitativo con valores de categorías de intervalos, se realizó test de Wil- coxon. Se consideraron diferencias significativas con $\mathrm{p} \leq$ 0,05 .

\section{Resultados}

1.Efecto de Ang-1-9 en la presión arterial sistólica (PAS): La administración crónica de Ang-(1-9) a las ratas LL-GB por un periodo de 2 semanas disminuyó significativamente la PAS respecto a las ratas GB sin tratamiento, en un $15 \%$ a la 1era sem post administración y manteniendo esta disminución (18\%) a la 2da sem post administración (Tabla1). Disminuciones similares en la PAS (18\%) se observaron en las ratas BN-GBAng-(1-9). El bloqueo del receptor MAS con A779, no modificó el efecto anti-hipertensivo de Ang-(1-9). Independiente del polimorfismo de la ECA, Ang-(1-9) disminuyó significativamente la PAS (Tabla1).

\begin{tabular}{|c|c|c|c|c|c|c|}
\hline & $\mathrm{n}$ & MC (gr) & MV (gr) & PAS i sem & PAS 5 sem & PAS 6 sem \\
\hline LL-Sham & 8 & $225 \pm 12$ & $0,68 \pm 0,01$ & $116 \pm 1$ & $110 \pm 1$ & $107 \pm 4$ \\
\hline $\begin{array}{l}\text { LL-GB } \\
\text { LL-GB }\end{array}$ & 8 & $248 \pm 14$ & $1,01 \pm 0,03^{\star}$ & $111 \pm 1$ & $161 \pm 4^{*}$ & $161 \pm 3^{*}$ \\
\hline Ang-(1-9) & 7 & $238 \pm 6$ & $0,84 \pm 0,04$ *\# & $111 \pm 4$ & $137 \pm 4^{\star} \#$ & $132 \pm 3^{\star} \#$ \\
\hline $\begin{array}{l}\text { LL-GB } \\
\text { Ang-(1-9)+A779 }\end{array}$ & 4 & $245 \pm 21$ & $0,83 \pm 0,10$ *\# & $114 \pm 1$ & $137 \pm 5^{\star \#}$ & $132 \pm 4^{\star \# \# ~}$ \\
\hline $\begin{array}{l}\text { BN-Sham } \\
\text { BN-GB }\end{array}$ & 8 & $\begin{array}{l}238 \pm 3 \\
241 \pm 8\end{array}$ & $\begin{array}{l}0,77 \pm 0,01 \& \\
1,0 \pm 0,02^{\star}\end{array}$ & $\begin{array}{r}109 \pm 1 \\
109 \pm 3\end{array}$ & $\begin{array}{l}111 \pm 2 \\
164 \pm 4^{*}\end{array}$ & $\begin{array}{l}110 \pm 2 \\
160 \pm 2^{*}\end{array}$ \\
\hline $\begin{array}{l}\text { BN-GB } \\
\text { Ang-(1-9) } \\
\text { BN-GB }\end{array}$ & 7 & $239 \pm 7$ & $0,91 \pm 0,04^{*}$ & $110 \pm 3$ & $134 \pm 5^{\star} \#$ & $135 \pm 7^{\star} \#$ \\
\hline & 4 & $211 \pm 21$ & $0,74 \pm 0,08 \# \&$ & $111 \pm 4$ & $142 \pm 7^{\star \#}$ & $133 \pm 7^{\star} \#$ \\
\hline
\end{tabular}

\section{Hipertrofia ventricular y cardiomiocitaria hiper-} tensiva: En condición de normotensión la MV de las ratas $\mathrm{BN}$ fue un $13 \%$ mayor que las ratas LL (Tabla1). Mientras que, el área y perímetro de los cardiomiocitos no mostró diferencias entre ambos polimorfismos $(203 \pm 8 \mathrm{v} / \mathrm{s} 180$ $\pm 9 \mathrm{~mm} 2,58,8 \pm 2 \mathrm{v} / \mathrm{s} \mathrm{55,8} \pm 2 \mathrm{~mm}$, Fig1 A, B y C). En respuesta al estímulo hipertensivo, la MV aumentó significativamente un $48 \%$ en las ratas LL-GB y un $30 \%$ en las ratas BN-GB (Tabla 1) versus sus controles normotensos, sin diferencias entre ambos polimorfismos. Al evaluar la hipertrofia cardiomiocitaria, se encontró un aumento significativo del 7\% del AC en las ratas LL-GB ( $\mathrm{p} \leq 0,01$, Fig1 A y B) y de un $23 \%$ en los animales BN-GB ( $\mathrm{p} \leq$ 0,001, Fig1 A y B) en relación a sus controles Sham respectivos sin diferencias entre ambos polimorfismos. En el PC sólo se observó un aumento del $9 \%$ en las ratas BNGB versus BN-Sham ( $p \leq 0,001$, Fig1 A y C). La adminis- 
Figura 1
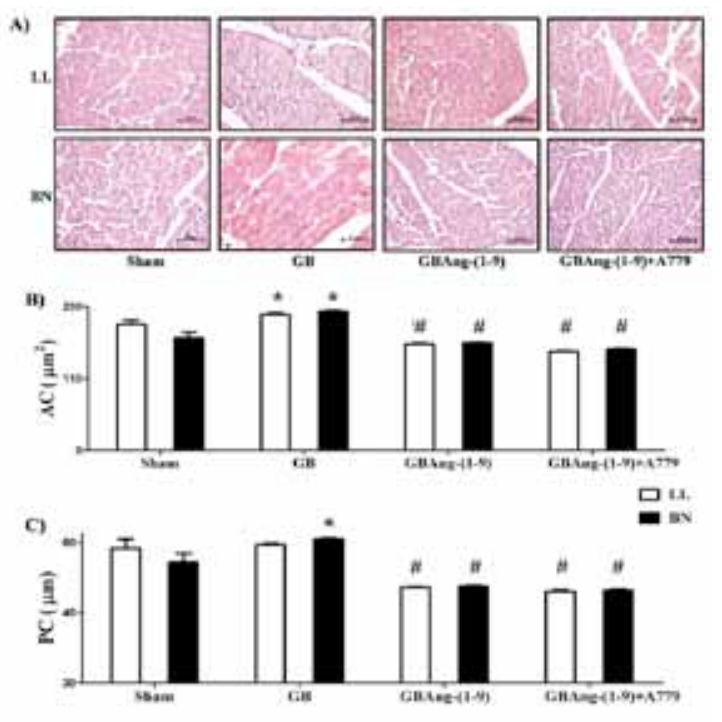

Figura 1. Efecto de Ang-(1-9) en el área de los cardiomiocitos de ratas homocigotos hipertensas. Abreviaturas: $A C=$ Área de los cardiomiocitos, Sham = ratas normotensas, $G B=$ ratas hiper tensas, GBAng-(1-9) = ratas hipertensas tratadas con Ang-(1-9), GBAng-(1-9)+A779 = ratas hipertensas tratados con Ang-(1-9) + A779, un antagonista del receptor de Ang-(1-7), LL = ratas Lewis con menor actividad de la ECA y $B N=$ ratas Brown Norway con mayor actividad de la ECA. A) cortes transversales de ventrículo de ratas hipertensas homocigotas para el polimorfismo de la ECA, teñidos con H-E. B) Evaluación del area de los cardiomiocitos. Los datos se graficaron según el promedio \pm SEM. $N=4-8, * p$ $\leq 0,05$ Sham y \# $p \leq 0,05$ GB; después de Kruskal-Wallis, seguido de un análisis de Mann-Whitney para comparación entre grupos.

tración de Ang-(1-9) disminuyó significativamente la MV en las ratas GB-LL (20\%, Tabla 1) en relación al grupo LL-GB que no recibió tratamiento con Ang-(1-9). Mientras que, en ratas BN-GB tratadas con Ang-(1-9) no se observaron variaciones significativas respecto a BN-GB (Tabla 1). La administración de Ang-(1-9) disminuyó el AC (22 y 23\%) tanto en ratas LL y BN GB, respectivamente versus las ratas $\mathrm{GB}$ sin tratamiento ( $\mathrm{p} \leq 0,0001$, Fig1 A y B). Igualmente disminuyó el PC (15 y 16) en los animales LL y BN GB versus sus controles hipertensos (Fig1 A y C). Sin observarse diferencias en el AC y PC entre ambos polimorfismos. La co-administración de Ang-(1-9) y A779, redujo la MV en las ratas LL y BN GB (18 y 26\%, respectiavmente, Tabla 1) respecto a sus controles GB. Igualmente, la co-administración de Ang-(1-9) y A779 también disminuyó el AC (28 y 27\%, respectivamente, $\mathrm{p}$ $\leq 0,0001$, Fig 1 A y B) y PC (17\% y $18 \%$, respectivamente, $\mathrm{p} \leq 0,0001$, Fig1 A y C) en las ratas LL y BN GB.
3. Hipertrofia Vascular: En ratas normotensas, el polimorfismo de la ECA no determinó diferencias en el ATM $(0,360 \pm 0,03 \mathrm{v} / \mathrm{s} 0,338 \pm 0,017 \mathrm{~mm} 2$, Fig 2 A y B $)$ y GTM $(64,78 \pm 3,34$ v/s $65,20 \pm 1,84 \mathrm{~mm}$, Fig2 A y C). En raFigura 2
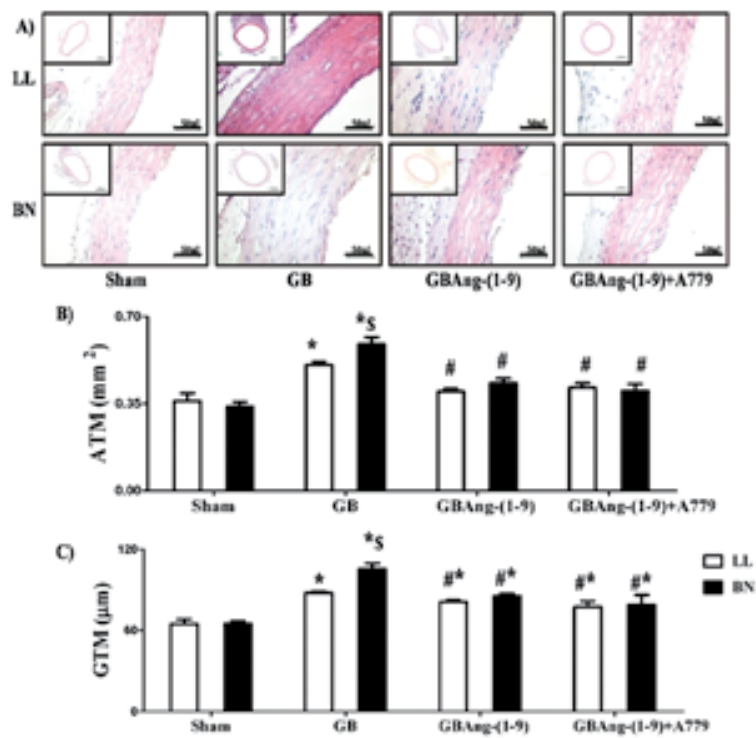

Figura 2. Efecto de Ang-(1-9) en la hipertrofia aórtica de ratas hipertensas homocigotas para el polimorfismo de la ECA. Abreviaturas: $A T M=$ Área de la túnica media, $G T M=$ Grosor de la túnica media, Sham = ratas normotensas, $G B=$ ratas hipertensas, GBAng-(1-9) = ratas hipertensas tratados con Ang-(1-9), GBAng$(1-9)+A 779=$ ratas hipertensas tratadas con Ang-(1-9) + A779, un antagonista del receptor de Ang-(1-7), $L L=$ ratas Lewis con menor actividad de la ECA y BN = ratas Brown Norway con mayor actividad de la ECA. A) Cortes transversales de aorta teñidos con H-E. B) Grafico del ATM. C) Grafico del GTM. Los resultados fueron graficados según el promedio \pm SEM. $N=4-8$. * $p \leq 0,05$ Sham, $\$ p \leq 0,05$ LL-GB y \# $p \leq 0,05$ GB; después de KruskalWallis, seguido de un análisis de Mann-Whitney para comparación entre grupos.

tas hipertensas, se encontró un aumento significativo del ATM de un 50\% en las ratas LL ( $p=0,002$, Fig2 A y B) $y$ de un $74 \%$ en las ratas BN ( $p=0,001$, Fig2 A y B). El GTM aumentó un $36 \%$ en las ratas LL ( $p=0,002$, Fig2 A y C) y un $62 \%$ en las ratas $\mathrm{BN}(\mathrm{p}=0,001$, Fig2 A y C) hipertensas versus sus controles sham. El polimorfismo de la ECA determinó que las ratas BN-GB muestran mayor $\operatorname{ATM}(0,589 \pm 0,027 \mathrm{v} / \mathrm{s} 0,505 \pm 0,013 \mathrm{~mm} 2, \mathrm{p}=0,022$, Fig2 A y B $)$ y GTM $(105,50 \pm 4,09$ v/s $87,85 \pm 0,86 \mathrm{~mm}$, $\mathrm{p}=0,001$, Fig2 A y C) que las ratas LL-GB. Ang-(1-9) disminuyó significativamente el ATM en un $21 \%$ ( $\mathrm{p}=$ 0,002 , Fig2 A y B) y el GTM un 7\% ( $p=0,002$, Fig2 A y C) en las ratas LL-GBAng-(1-9) v/s LL-GB. Igualmente 
en ratas BN-GB Ang-(1-9), disminuyó el ATM un 26\% ( $p=0,001$ Fig2 A y B) y el GTM un 19\% ( $p=0,001$, Fig2 A y C), versus BN hipertensas. El polimorfismo de la ECA no determinó una respuesta diferente en el ATM y GTM (Fig.2). La co-administración de Ang-(1-9) y A779, disminuyó significativamente en las ratas LL y BN GB, el ATM $(18 \%, p=0,01$ y $32 \%, p=0,006$ respectivamente; Fig2 A y B) y el GTM (12\%, p = 0,01 y 25\%, p = 0,006 respectivamente, Fig2 A y C) en comparación a los animales LLy BN-GB sin tratamiento.

Figura 3
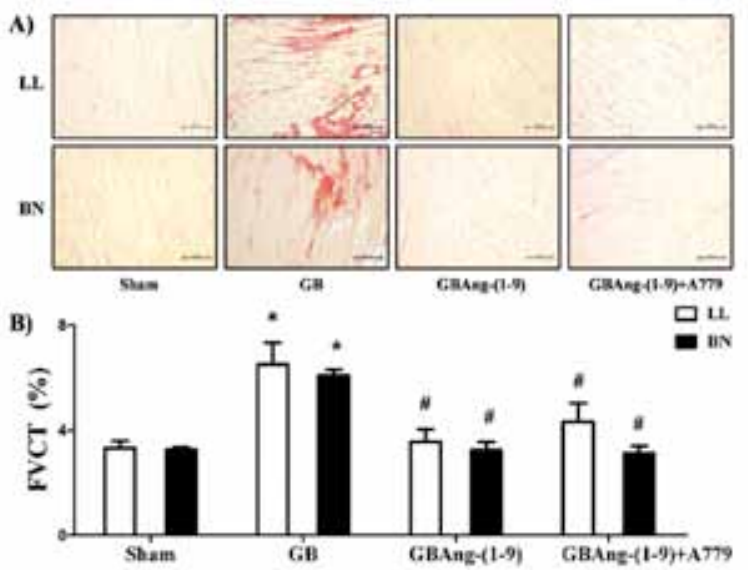

Figura 3. Efecto de Ang-(1-9) en el porcentaje de colágeno miocárdico en ratas hipertensas homocigotos para el polimorfismo de la ECA. Abreviatura: $F V C T=$ Facción volumétrica de colágeno total, Sham = ratas normotensas, $G B=$ ratas hipertensas, GBAng-(1-9) $=$ ratas hipertensas tratadas con Ang-(1-9), GBAng-(1-9)+A779 = ratas hipertensas tratadas con Ang-(1-9) $+A 779$, un antagonista del receptor de Ang-(1-7), LL = ratas Lewis con menor actividad de la ECA y BN = ratas Brown Norway con mayor actividad de la ECA. A) Cortes de la zona miocárdica del VI teñidos con rojo picrosirio. B) grafico de la FVCT entre los modelos experimentales. Los datos fueron graficados según el promedio \pm SEM, $N=$ $4-8$, * $p \leq 0,05$ Sham y \# $p \leq 0,05$ GB, después de Kruskal-Wallis, seguido de un análisis de Mann-Whitney para comparación entre grupos.

4. Fibrosis miocárdica: En ratas normotensas el polimorfismo de la ECA no determinó diferencias en la FVCT $(3,33 \pm 0,26 \mathrm{v} / \mathrm{s} 3,28 \pm 0,08, \%$, Fig3 A y B $)$ entre las ratas LLy BN Sham, respectivamente. En respuesta al estímulo hipertensivo las ratas LL y BN GB mostraron un aumento estadísticamente significativo de la FVCT $(6,53 \pm 0,83 \mathrm{v} / \mathrm{s}$ $3,33 \pm 0,26 \%, \mathrm{p} \leq 0,0001$ y $6,10 \pm 0,22 \mathrm{v} / \mathrm{s} 3,28 \pm 0,08$ $\%, \mathrm{p}=0,001$, respectivamente, Fig3 A y B) versus los ani- males normotensos LL y BN. El polimorfismo de la ECA no determinó diferencias significativas en el contenido de colágeno entre las ratas LL y BN GB (Fig3 A y B). La administración de Ang-(1-9) disminuyó un 45\% la FVCT en las ratas LL-GB ( $p=0,008$, Fig3 A y B) versus LL-GB sin tratamiento. Igualmente, en las ratas $\mathrm{BN}-\mathrm{GB}$ la Ang(1-9) disminuyó un 47\% la FVCT ( $p=0,002$, Fig3 A y B) respecto a las ratas BN-GB sin tratamiento. La co-administración de Ang-(1-9) y A779 en las ratas hipertensas LL y BN, no modificó el efecto anti-fibrótico de Ang-(1-9) y de regresar el contenido de colágeno total respecto a los animales GB que recibieron sólo Ang-(1-9), observándose en las ratas LL y BN GB tratadas con Ang-(1-9) y A779 una disminución significativa de la FVCT (34\%, $\mathrm{p}=0,05$ y $48 \%, p=0,01$ respectivamente, Fig3 A y B) versus los animales LL y BN GB sin tratamiento. El polimorfismo de la ECA no determinó una respuesta diferencial al tratamiento en la FVCT (Fig3 A y B), entre las ratas LL y BN hipertensos.

Figura 4

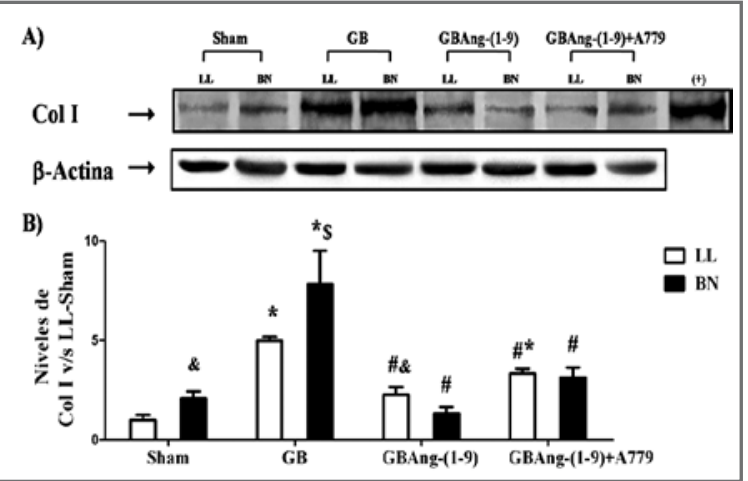

Figura 4. Efecto de Ang-(1-9) en los niveles proteicos de colágeno I en la pared aórtica en ratas hipertensas homocigotos para el polimorfismo de la ECA. Abreviatura: Col I = colágeno I, Sham = ratas normotensas, $G B=$ ratas hipertensas, GBAng-(1-9) = ratas hipertensas tratadas con Ang-(1-9), GBAng-(1-9)+A779 = ratas hipertensas tratadas con Ang-(1-9) + A779, un antagonista del receptor de Ang-(1-7), LL = ratas Lewis con menor actividad de la ECA y niveles de Ang II y $B N=$ ratas Brown Norway con mayor actividad de la ECA y niveles de Ang II. A) Diferencias en los niveles proteicos de Col I entre los 4 grupos experimentales. B) Gráfico de los niveles de Col I v/s LL-Sham. Los datos fueron graficados según el promedio \pm SEM, $N=4-8$, * $p \leq 0,05$ Sham, $\& p \leq 0,05$ LL-Sham, $\$ p \leq 0,05$ LL-GB y \# $p \leq 0,05$ GB, después de Kruskal-Wallis, seguido de un análisis de Mann-Whitney para comparación entre grupos.

5. Fibrosis vascular: En condición de normotensión los niveles proteicos de $\mathrm{Col}$ I fueron significativamente mayores en las ratas BN versus LL-Sham $(2,08 \pm 0,37 \mathrm{v} / \mathrm{s} 1$ $\pm 0,25, p=0,05$, Fig4 A y B). Frente a una condición de 
hipertensión, el nivel proteico de Col I en las ratas LL y BN GB aumentó significativamente respecto a sus controles normotensos LL y BN $(4,98 \pm 0,20 \mathrm{v} / \mathrm{s} 1 \pm 0,25$, p $=0,001$ y 7,83 $\pm 1 \mathrm{v} / \mathrm{s} 2,08 \pm 0,37, \mathrm{p} \leq 0,0001$ respectivamente, Fig4 A y B). El polimorfismo de la ECA determinó mayores niveles proteicos de Col I en las ratas BN-GB v/s LL-GB $(7,83 \pm 1$ v/s 4,98 $\pm 0,20, p=0,011$, Fig4 A y B). La administración crónico de Ang-(1-9) en los animales hipertensos, disminuyó significativamente los niveles proteicos de Col I en las ratas LL-GB, versus las ratas LLGB sin tratamiento (Col I: 2,28 $\pm 0,38 \mathrm{v} / \mathrm{s} 4,98 \pm 0,20, \mathrm{p}$ $=0,001$, Fig. 4). Igual comportamiento se observó en las ratas BN-GB tratadas con Ang-(1-9) (Col I: 1,34 \pm 0,31 v/s 7,83 $\pm 1, p \leq 0,0001$, Fig4 A y B) respecto a su control hipertensos. El efecto anti-fibrótico de Ang-(1-9) no se alteró por la co-administración de A779, observándose en las ratas LL y BN GB una disminución significativa de los niveles proteicos de Col I (33\%, p = 0,01 y 54\%, p = 0,028, respectivamente, Fig4 A y B). El polimorfismo de la ECA, no determinó una respuesta fibrótica diferencial (Fig4 A y B) entre las ratas LL y BN GB con tratamiento.

6. Inflamación miocárdica: Las ratas normotensas LL y BN no mostraron diferencias en el número de células infiltradas positivas para ED1 (Tabla 2). Mientras que, en animales hipertensos se encontraron mayores niveles de Céls (+) para ED1 en comparación con la ratas Sham, donde a los grupos LL y BN GB se les asignó un valor de 2 según escala semi-cuantitativa. Estableciendo que los animales presentaron un número moderado de Céls (+) para ED1 distribuidos homogéneamente en el VI, con un rango de 20 a 39 Céls (+) ED1, aproximadamente (2 v/s 0 valor asignado, LL: $\mathrm{p}=0,019$ y BN: $\mathrm{p}=0,038$, Tabla 2). El análisis semi-cuantitativo no encontró diferencias asociadas al polimorfismo de la ECA, entre las ratas hipertensas LL y BN. El efecto de Ang-(1-9) en las ratas LL y BN GB disminuyó el número de Céls (+) para ED1, según la escala semi-cuantitativa, en relación con los animales hipertensos sin tratamiento $(1 \mathrm{v} / \mathrm{s} 2$ valor asignado, LL: $\mathrm{p}=$ 0,037 y BN: $p=0,05$, Tabla 2). La co-administración de Ang-(1-9) y A779 no modificó el efecto anti-inflamatorio de Ang-(1-9) en las ratas hipertensas LL y BN (Tabla 2).

7. Inflamación en la pared aórtica: En ratas normotensas, se observó un aumento del $60 \%$ en la razón entre las células totales positivas para ED1 y el área del anillo aórtico (ED1/A) en las ratas $\mathrm{BN}$ respecto a los animales LL ( $p=0,005$, Fig 5 A y B). La hipertensión aumentó sig-

\begin{tabular}{|c|c|c|c|c|}
\hline Modelo & n & Valor asignado & $\begin{array}{l}\text { Rango de Cels } \\
\qquad(+) \text { ED-1 }\end{array}$ & Significando \\
\hline LL-Sham & 7 & 0 & 0 & Sin presencia de Céls (+) ED-1 \\
\hline Ш-GB & 7 & 2 & 20 a 39 & $\begin{array}{l}\text { Número moderado de Céls (+) ED-1 } \\
\text { distribuidos homogéneamente en VI }\end{array}$ \\
\hline \multicolumn{5}{|l|}{ LL-GB } \\
\hline Ang-(1-9) & 7 & 1 & 0 a 19 & $\begin{array}{l}\text { Bajo número de Céls (+) ED-1 } \\
\text { aislados en VI }\end{array}$ \\
\hline \multicolumn{5}{|l|}{ LL-GB } \\
\hline Ang-(1-9)+A779 & 4 & 1 & 0 a 19 & $\begin{array}{l}\text { Bajo número de Céls (+) ED-1 } \\
\text { aislados en VI }\end{array}$ \\
\hline BN-Sham & 9 & 0 & 0 & Sin presencia de Céls (+) ED-1 \\
\hline BN-GB & 7 & 2 & 20 a 39 & $\begin{array}{l}\text { Número moderado de Céls (+) ED1 } \\
\text { distribuidos homogéneamente en VI }\end{array}$ \\
\hline \multicolumn{5}{|l|}{ BN-GB } \\
\hline Ang-(1-9) & 7 & 1 & 0 a 19 & $\begin{array}{l}\text { Bajo número de Céls (+) ED1 } \\
\text { aislados en VI }\end{array}$ \\
\hline \multicolumn{5}{|l|}{ BN-GB } \\
\hline Ang-(1-9)+A779 & 4 & 1 & 0 a 19 & $\begin{array}{l}\text { Bajo número de Céls (+) ED1 } \\
\text { aislados en VI }\end{array}$ \\
\hline
\end{tabular}

nificativamente la razón ED1/A en las ratas LL $(918 \pm 117$ v/s 246 $\pm 27 \mathrm{~N}^{\circ}$ células/lmm2, p $\leq 0,0001$, Fig 5 A y B) y $\mathrm{BN}\left(1422 \pm 192 \mathrm{v} / \mathrm{s} 394 \pm 32 \mathrm{~N}^{\circ}\right.$ células/mm2, $\mathrm{p}=0,001$, Fig 5 A y B) con respecto a los controles LL y BN normo-

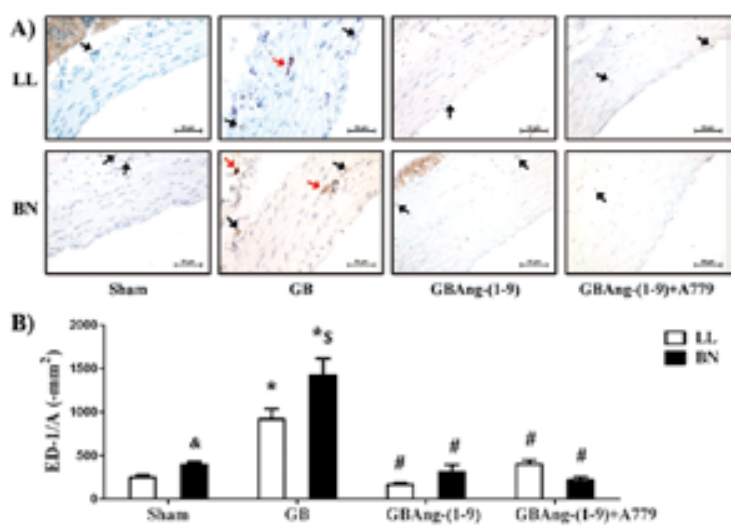

Figura 5. Efecto de Ang-(1-9) en la infiltración de macrófagos a nivel vascular en ratas hipertensas. Abreviaturas: Céls $T(+)$ ED-1/A = Razón entre el número de células totales positivas para ED-1 y el área de anillo aórtico, Sham = grupos normotensos, GB = grupos hipertensos, GBAng-(1-9) = grupos hipertensos tratados con Ang-(1-9), GBAng-(1-9)+A779 = grupos hipertensos tratados con Ang-(1-9) + A779, LL = ratas Lewis con menor actividad de la ECA y niveles de Ang II, BN = ratas Brown Norway que presentan mayor actividad de la ECA y niveles de Ang II, > = céls (+) ED-1 y $>=$ cúmulo de céls (+) ED-1. A) Inmunohistiquímica de cortes de la pared aórtica revelados con Diaminobenzidina y contratinción con hematoxilina. Se graficó la razón Cels $T(+)$ ED-1/A (B) entre los modelos experimentales. Los datos fueron graficados según el promedio $\pm S E M, N=4-8, \& p \leq 0,05$ LL-Sham, * $p \leq 0,05$ Sham, $\$ p \leq 0,05$ LL-GB y \# $p \leq 0,05$ GB, después de Kruskal-Wallis, seguido de un análisis de Mann-Whitney para comparación entre grupos. 
tensos. El polimorfismo de la ECA determinó un aumento de un $55 \%$ en la razón ED1/A de las ratas BN-GB v/s LL-GB ( $p=0,029$, Fig 5 A y B). Las administración de Ang-(1-9) a la 4ta sem post-cirugía GB, disminuyó significativamente la razón ED1/A un 82\% en las ratas LL-GB ( $p \leq 0,0001$, Fig 5 A y B) y un $78 \%$ en las ratas BN-GB ( $p=0,001$, Fig 5 A y B) respecto a las ratas hipertensas LL y BN sin tratamiento. La co-administración de Ang(1-9) + A779 mantuvo el efecto anti-inflamatorio de Ang(1-9) disminuyendo significativamente en la razón ED1/A en los modelos LL y BN GBAng-(1-9)+A779 (57\%, p $=0,012$ y $85 \%, p=0,004$ respectivamente, Fig5 A y B) versus los grupos LL y BN GB. No se encontró variación en la razón ED1/A asociadas a los niveles genéticamente determinados de ECA y Ang II entre las ratas LL y BN tratadas con Ang-(1-9) o con la co-administración de Ang(1-9) y A779.

\section{Discusión}

Los principales resultados de este estudio fueron, el polimorfismo de la ECA con mayores niveles de ECA y Ang II determinó: a) en ratas normotensas e hipertensas mayor fibrosis e inflamación en la pared aórtica y b) En ratas hipertensas mayor hipertrofia miocitaria. La administración crónica de Ang-(1-9) a ratas hipertensas disminuyó significativamente a) La PAS y el RMCV en órganos blanco como corazón y aorta, b) la hipertrofia c) la fibrosis y d) la inflamación. El polimorfismo de la ECA no determinó una respuesta diferencial al tratamiento con Ang-(1-9).

1. Polimorfismo de la ECA y RMCV: En condiciones de normotensión, el polimorfismo de la ECA determinó un aumento de $\mathrm{MV}$, niveles proteicos de $\mathrm{Col}$ I e infiltración de macrófagos en la pared aórtica de ratas $\mathrm{BN}$ versus LL. Estos resultados son apoyados por resultados descritos por Ocaranza et al., (2008) ${ }^{33}$ que establecieron un mayor remodelamiento molecular expresado como mayores niveles de ARNm y proteína de TGF $\beta 1$, PAI1 y MCP-1 en la pared aórtica de las ratas $\mathrm{BN}$ respecto a las ratas LL. Además, los niveles de expresión genética y proteíca para ECA fueron significativamente mayores, mientras que los de ECA2 fueron menores en los animales BN versus LL. Recientemente, resultados del laboratorio han mostrado, que existe un mayor remodelamiento de la pared aórtica en ratas $\mathrm{BN}$ normotensas con respecto a las ratas LL, ${ }^{29}$ asociado a un mayor estrés oxidativo por aumen- to de la actividad de la NADP(H) y mayor producción de radicales $\mathrm{O} 2-.34$

\section{Polimorfismo de la ECA y su relación con HTA y su}

RMCV: Diversos estudios han examinado la asociación entre el genotipo de la ECA y alteraciones cardiacas como la HTA, restenosis arterial coronaria, etc, entre otras. ${ }^{35}$ En general, los resultados de estos estudios clínicos y poblacionales han indicado que el polimorfismo del gen de la ECA parece no contribuir al desarrollo de HTA en humanos. ${ }^{36}$ Nuestros resultados no muestran diferencias significativas en la PAS entre las ratas hipertensas BN y LL ( $N=8$ y 6 semanas de ensayo). Por el contrario, resultados previos del laboratorio ${ }^{20}$ han mostrado que el polimorfismo que determina mayores niveles de ECA y Ang II, muestra mayor PAS en ratas sometidas a un estímulo hipertensivo. Estas diferencias pudieron ser encontradas en un tamaño muestral mayor ( $\mathrm{N}=19-41$ animales) y a tiempos de exposición al estímulo hipertensivo de 9 semanas. Igualmente, en humanos los estudios que han encontrado una correlación entre hipertensión y el polimorfismo DD han tenido que reclutar muestras poblacionales grandes. ${ }^{37}$ Además, se ha mostrado que el polimorfismo del gen de la ECA que determina mayores niveles de ECA y Ang II podría inducir respuestas o riesgos diferentes en relación con estímulos fisiológicos y patológicos. Al respecto, Challah et al (1998) ${ }^{38}$ encontraron que ratas con mayor actividad de la ECA (ratas BN) desarrollaron mayor proliferación de la neoíntima por daño cardíaco en comparación con ratas con menor actividad de al ECA. Nuestros resultados muestran que frente al estímulo hipertensivo las ratas BN-GB mostraron, un aumento de la hipertrofia, fibrosis e inflamación a nivel de la pared aórtica, con respecto las ratas hipertensas LL-GB. Estos resultados apuntan a que mayores niveles de ECA y Ang II se asocian a un mayor remodelamiento vascular secundario a la HTA. Es conocido que la Ang II induce hipertrofia de la pared aórtica por medio de un incremento de la PA y por lo tanto de estímulos mecánicos que gatillan una mayor expresión de PDGF-A, que podría actuar como mediador de la respuesta hipertrófica vascular. ${ }^{39}$ Además varios estudios han demostrado que Ang II induce fibrosis e inflamación vascular. ${ }^{40,41}$ Estos efectos son mediante una compleja interacción de vías de señalización intracelular que involucran Fosfolipasas, factores de transcripción de genes y Activación de tirosinas kinasas. ${ }^{42}$ 
3. Efecto de Ang-(1-9) en la hipertensión arterial: Aunque la Ang-(1-9) está presente en plasmas humanos $\mathrm{y}$ de $\operatorname{ratas}^{19} \mathrm{y}$ sus niveles circulantes están aumentados en condiciones patológicas como en el remodelamiento temprano post IAM19 o en animales tratados con inhibidores de la ECA,10 sus efectos biológicos recién han comenzado a describirse. ${ }^{16}$ Los antecedentes disponibles a la fecha en relación al compromiso de componentes de la vía paralela del SRA en la HTA están concentrados en la ECA-2 y la Ang-(1-7), ${ }^{43}$ mostraron en ratas espontáneamente hipertensas (SHRSP) que tienen menor expresión génica y proteica de ECA-2 en riñón, el rol de la ECA-2 en la patogénesis de la HTA. Específicamente generaron ratas transgénicas SHRSP que sobreexpresaron la ECA-2 humana en células musculares lisas vasculares (SHRSPACE2). Las ratas SHRSP-ACE2 mostraron aumento en los niveles de Ang-(1-7), disminución de la presión sanguínea, menor respuesta vasoconstrictora al estímulo de Ang II y mejoría en la función endotelial comparado con las ratas SHRSP. ${ }^{43}$ Por su parte la Ang-(1-7) ha mostrado ser un importante regulador de la función cardiovascular y renal promoviendo la vasodilatación, apoptosis y efecto anti-proliferativo.14,15 La Ang-(1-7), a través de su receptor, el producto del oncogén Mas ${ }^{44}$ estimularía la óxido nítrico sintetasa (NOS) y contrarrestaría las acciones de Ang II. El efecto de la Ang-(1-7) también podría ser vía su unión al receptor tipo 2 de Ang II, aumentando la unión de bradikinina a su receptor B2.45 Ang-(1-7) se degrada a péptidos inactivos por acción de la ECA. Por lo tanto, la inhibición de la ECA podría aumentar los niveles de Ang-(1-7) y simultáneamente disminuir los de Ang II. Ya que Ang-(1-7) es un potente péptido vasodilatador, su acción contrarrestaría el efecto vasopresor de Ang II. Por lo tanto, la ECA-2 intervendría en la mantención del tono vascular, contrarregulando los efectos derivados de la ECA. ${ }^{46}$ Los resultados del estudio muestran que la administracióon crónica de Ang-(1-9) disminuye la PAS tanto en las ratas hipertensas LL y BN. Este efecto se mantuvo en las ratas tratadas con Ang-(1-9)+A779, mostrando que el efecto antihipertensivo de Ang-(1-9) es directo y no es mediado por la tranmsformación de Ang-(1-9) a Ang(1-7). Se conoce que Ang-(1-9) favorece la unión de la Bradikininas (BK) a su receptor B2, probablemente por cambios conformacionales en el complejo ECA- receptor B2 ${ }^{47}$ beneficiando las acciones de la BK al estimular la vasodilatación, la formación de $\mathrm{NO}$ y de prostaciclinas. ${ }^{48}$
4. Efecto de Ang-(1-9) en el RMCV hipertensivo: Recientemente, hemos publicado que Ang-(1-9) presenta efectos biológicos al prevenir la hipertrofia miocárdica tanto in vivo in vitro. ${ }^{16}$ Además, resultados también recientes del laboratorio mostraron que ratas sometidas a un estimulo hipertensivo por sobrecarga de presión presentaron menores niveles de ECA2 y Ang-(1-9), lo que se asociado a un mayor remodelamiento de la pared aórtica. ${ }^{17}$ Nuestros resultados muestran que el tratamiento crónico con Ang-(19) de ratas hipertensas LL y BN con Ang-(1-9) disminuyó la MV, la hipertrofia de la pared aórtica y de los cardiomiocitos, además de la fibrosis miocárdica, contenido de colágeno I e inflamación en la pared aórtica. Estudios realizados en un modelo experimental post-IAM, determinó un aumento de la actividad enzimática de la ECA y de los niveles de Ang II, junto a una disminución de la actividad enzimática de la ECA2 y de los niveles de Ang-(1-9),19 estos cambios, favorecieron la fibrosis miocárdica en el área no infartada y el desarrollo de hipertrofia ventricular patológica.19 Es conocido que el Enalapril disminuye el remodelamiento cardíaco post-IAM, efecto que se asocia a la disminución de la actividad de ECA y de los niveles de Ang II, en conjunto con un aumento en la actividad de la ECA2 y de los niveles de Ang-(1-9).16 Por largo tiempo, la atención se ha enfocado en la Ang-(1-7), y como este péptido contrarresta los efectos de Ang II, a través de la estimulación de la Oxido Nítrico Sintasa y de los niveles de Bks.45 Estudios en ratas que recibieron una administración crónica de Ang-(1-7), comprobaron su capacidad para prevenir el remodelamiento cardíaco inducido por Ang II y la fibrosis cardíaca asociada a la hipertensión. ${ }^{49}$ Investigaciones sugieren que la unión de Ang-(1-7) al receptor específico en fibroblastos cardíacos inicia efectos anti-fibróticos y anti-tróficos. ${ }^{50}$

Existe solo un reporte recientemente publicado por Kramkowski et al., $2010{ }^{51}$ respecto a efectos adversos de la administración de Ang-(1-9) a ratas a dosis de 1893 ng kg-1 min-1 por un periodo de 65 minutos. Los autores observaron un efecto protrombótico de Ang-(1-9) probablemente mediado por mayor formación de fibrina, activación de plaquetas o por la convesión de Ang-(1-9) a Ang II debido a la hidrólisis por enzimas presentes en la sangre, como también las presentes y liberadas por las plaquetas. Es importante hacer notar que estos efectos adversos no han sido descritos previamente y fueron observados en una dosis tres veces mayor que la dosis usada en nuestros ensayos in vivo de ratas con hipertensión establecida. Has- 
ta la fecha se han establecido dos ejes primordiales en el balance fisiológico del SRA, el eje vasoconstrictor, ECAAng II-RAT1 y el eje vasodilatador, ECA2-Ang-(1-7)receptor MAS, ${ }^{52} \sin$ tomar importancia al posible rol del eje ECA2-Ang1-9. Nuestros resultados apoyan el concepto que Ang-(1-9) tiene un efecto antihipertensivo junto con ser eficaz en disminuir o regresar el remodelamiento cardiovascular y daño en órganos blanco secundario a la hipertensión.

Las principales limitaciones de este estudio fueron que no se determinaron los niveles de las angiotensinas. Además, se desconocen las posibles vías de transducción de señales activadas por Ang-(1-9), que pudieran contribuir a la disminución de la HTA y del remodelamiento cardio- vascular). La proyección de este trabajo es que Ang-(1-9) podría constituir un nuevo modelo de fármaco peptídico para el tratamiento de la HTA y su RMCV asociado. Además, el conocimiento del receptor de Ang-(1-9) permitirá modelar y sintetizar péptidos miméticos de Ang-(1-9) más eficaces y seguros en el tratamiento de la hipertensión y del daño residual en órganos blanco.

En conclusión, Ang-(1-9) disminuye eficazmente la HTA y el remodelamiento cardiovascular secundario, independientemente del polimorfismo del gen de la ECA. Este efecto es directo ya que no fue mediado por Ang-(1-7).

\section{AGRADECIMIENTOS}

Este trabajo fue financiado Fondecyt 1100874 


\section{Referencias}

1. ORGANIZACIÓN MUNDIAL DE LA SALUD. Programa general. Prevención de las enfermedades crónicas: Una inversión vital. Public Health Agency of Canada, 2005.

2. MINISTERIO DE SALUD, Encuesta Nacional de Salud de Chile 2003; 1:95-121.

3. MOLINER J.R, DOMÍNGUEZ M, GONZÁLEZ M.C, ALFARO G, CRESPO J, RODRÍGUEZ M, et al. Hipertensión Arterial. Guías clínicas 2004; 4:1-10.

4. TOUYZR. Intracellular mechanisms involved in vascular remodelling of resistance arteries in hypertension: role of angiotensin II. Exp Physiol. 2005; 90: 449-455.

5. INTENGAN H, SCHIFFRIN E. STRUCTURE AND MECHANICAL Properties of Resistance Arteries in Hypertension: Role of Adhesion Molecules and Extracellular Matrix Determinants Hypertension 2000; 36:312-318.

6. BERK B, FUJIWARA K, LEHOUX S. ECM remodeling in hypertensive heart disease. J. Clin. Invest. 2007; 117: 568-575.

7. MESSERLI F, WILLIAMS B, RITZ E. Essential hypertension, Lancet 2007; 370:591-603

8. AHUMADAL, BORJAH, GEREA, JALIL J,LUCO L, MONTERO J, et al. MINISTERIO DE SALUD. Guía Clínica Hipertensión Arterial Primaria o Esencial en personas de 15 años y más. 1ra Ed. Santiago: Minsal, Chile, 2006.

9. TIPNIS S, HOOPER N, HYDE R, KARRAN E, CHRISTIE G, TURNER AJ, et al. A human homolog of angiotensin converting enzyme. Cloning and functional expression as a captopril-insensitive cardoxypeptidase. J. Biol. Chem 2000; 275: 33238-33243.

10. DONOGHUE M, HSIEH F, BARONAS E, GODBOUT K GOSSELIN M, STAGLIANO N, et al. A novel angiotensinconverting enzyme related carboxypeptidase (ACE2) converts angiotensin I to angiotensin 1-9. Circ. Res 2000; 87: e1-e9.

11. VICKERS CH, HALES P, KAUSHIK H, DICK L, GAVIN J, TANG J, et al. Hydrolisis of biological peptides by human angiotensin-converting enzyme-related carboxypeptidase (ACE2). J. Biol. Chem 2002; 277: 14838-14843.

12. RICE G, THOMAS D, GRANT P, TURNER A, HOOPER N
Evaluation of angiotensin-converting enzyme (ACE), its homologue ACE2 and neprilysin in angiotensin peptide metabolism. Biochem J 2004; 383:45-51.

13. SANTOS R, CASTRO C, GAVA E, PINHEIRO S, ALMEIDA A, PAULA RD, et al. Impairment of in vitro and in vivo heart function in angiotensin-(1-7) receptor MAS knockout mice. Hypertension 2006; 47: 996-1002.

14. FERRARIO C. Angiotensin-converting enzyme 2 and angiotensin-(1-7): an evolving story in cardiovascular regulation. Hypertension 2006; 47: 515-521

15. OCARANZA M, LAVANDERO S, JALIL J, MOYA J, PINTO M, NOVOA U, et al. Angiotensin-(1-9) regulates cardiac hypertrophy in vivo and in Vitro. Journal of Hypertension 2010; 28:1054-1064.

16. OCARANZA M, MOYA J, PINTO M, ESCUDEROS N, VALENZUELA F, VARAS M, et al. Menores niveles tisulares de la enzima convertidora de angiotensina I homóloga (ECA-2) y angiotensina-(1-9) están asociados a mayor remodelamiento de la pared aórtica de las ratas hipertensas. Rev Chil Cardiol 2010; 29:69-82.

17. LAGOS T, ESCUDE N, NOVOA U, GODOY I, CHIONG M, et al. Angitensina 1-9 previene la hipertensión arterial y el remodelamiento de la pared aortica inducido por Angiotensina II. Rev Chil Cardiol, Libro resúmenes 2009; 38:333-334.

18. GONZÁLEZ L, CONTRERAS A, NOVOA U, GODOY I, CHIONG M, et al. Evidencias in vitro e in vivo de la acción antihipertrófica de Angitensina 1-9 en el cardiomiocito. Rev Chil Cardiol, Libro resúmenes 2009; 38:299-300.

19. OCARANZA M, GODOY I, JALIL J, VARAS M, PATRICIA COLLANTES, PINTO M, et al. Enalalpril attenuates downregulation of angiotensin-converrung enzyme 2 in the late phase of ventricular dysfunction in myocardial infracted rat. Hypertension 2006; 48:572-578.

20. OCARANZA M, PIDDO A, FAÚNDEZ P, LAVANDERO S, JALIL, J. Angiotensin I-converting enzyme gene polymorphism influences chronic hypertensive response in the rat Goldblatt model. J. Hypertension 2002; 20:413-420.

21. OLIVERI C, OCARANZA M, CAMPOS X, LAVANDERO S JALIL J.Angiotensin I-converting enzyme modulates neutral endopeptidase activity in the rat. Hypertension 2001; 38:650-654 .

22. GOLDBLATT H, LYNCH J, HANZAL R, SUMMERVILLE 
W. Studies on experimental hypertension: I. the production of persistent elevation of systolic blood pressure by means of renal ischemia. J Exp Med 1934; 59:347-379.

23. LARA L, BICA R, SENA S, CORREA J, MARQUES-FERNANDES M, LOPES AG, et al. Angiotensin-(1-7) reverts the stimulatory effect of angiotensin II on the proximal tubule $\mathrm{Na}$ (+)-ATPase activity via a A779-sensitive receptor. Regul Pept 2002; 103:17-22.

24. OCARANZA M, GODOY I, JALIL J, VARAS M, PATRICIA COLLANTES P, PINTO M, et al. Enalalpril attenuates downregulation of angiotensin-converrung enzyme 2 in the late phase of ventricular dysfunction in myocardial infracted rat. Hypertension 2006; 48:572-578.

25. IGASE M, STRAWN W, GALLAGHER P, GEARY R, FERRARIO C. Angiotensin II AT1 receptors regulate ACE2 and angiotensin-(1-7) expression in the aorta of spontaneously hypertensive rats. Am J Physiol Heart Circ Physiol 2005; 289:H1013H1019.

26. NAKAMURA K, FUSHIMI K, KOUCHI H, MIHARA K, MIYAZAKI M. Inhibitory effects of antioxidants on neonatal rat cardiac myocyte hypertrophy induced by tumor necrosis factor-a and angiotensin II. Circulation 1998; 98:794-799.

27. JALIL J, JANICKI J, PICK R, WEBER K. Coronary vascular remodeling and myocardial fibrosis in the rat with renovascular hypertension. Response to captopril. Am J Hypertens 1991; 4:51-55.

28. RIVERA P, OCARANZA M, LAVANDERO S, JALIL J. RHO KINASE Activation and Gene Expression Related to Vascular Remodeling in Normotensive Rats With High Angiotensin I Converting Enzyme Levels. Hypertension 2007; 50:792-798.

29. BRADFORD M. A rapid and sensitive method for the cuantitation of microgram cuantities of protein utilizing the principle of protein-dye binding. Anal. Biochem 1976; 72:248-254.

30. SALVADÓ M, MARTÍNEZ S, ÁLVARO T, BARBERA M, RISA R, et al. Técnicas de desenmascaramiento antigénico en inmunohistoquímica. Rev Esp Patol 2001; 34:255-260.

31. LIU J, YANG F, YANG X, JANKOWSKI M, PAGANO P. NAD (P)H Oxidase Mediates Angiotensin II-Induced Vascular Macrophage Infiltration and Medial Hypertrophy. Arterioscler Thromb Vasc Biol 2003; 23:776-782.
32. ALLAN A, FENNING A, LEVICK S, HOEY A, BROWN L. Reversal of cardiac dysfunction by selective ET-A receptor antagonism. British J Pharmacol 2005; 146:846-853.

33. OCARANZA M, MOYAJ, VALENZUELAF, VARAS M, JALIL J. Menor expresión de la enzima convertidora de Angiotensinal homóloga (ECA2) se asocia a mayor remodelamiento de la pared aórtica de ratas con niveles genéticamente elevados de Angiotensina II. Rev Chil Cardiol 2008; 27:167-171.

34. JALIL J, PÉREZA, OCARANZA M, BARGETTO J, GALAZ A, LAVANDERO $S$, et al. Increased aortic NADPH oxidase activity in rats with genetically high angiotensin-converting enzyme levels. Hypertension 2005, 46:1362-1367.

35. LACHURIÉ M, AZIZI M, GUYENE T, ALHENC-GELAS F, MÉNARD J. Angiotensin-converting enzyme gene polymorphism has no influence on the circulating renin-angiotensinaldosterone system or blood pressure in normotensive subjects. Circulation 1995; 91: 2933-2342.

36. BERGE K, BERG K. No effect of insertion/deletion polymorphism at the ACE locus on normal blood pressure level or variability. Clin Genet 1994; 45:169-174.

37. O'DONNELL C, LINDPAINTNER K, LARSON M, RAO V, ORDOVAS J, SCHAEFER EJ, et al. Evidence for association and genetic linkage of the angiotensin-converting enzyme locus with hypertension and blood pressure in men but not women in the Framingham Heart Study. Circulation 1998; 97:1766-1772.

38. CHALLAH M, VILLARD E, PHILIPPE M, RIBADEAUDUMAS A, GIRAUDEAU B, JANIAK P, et al. Angiotensin I-converting enzyme genotype influences arterial response to injury in normotensive rats. Arterioscler Thromb Vasc Biol 1998;182:235-243.

39. PARKER S, WADE S, Prewitt R. Pressure Mediates Angiotensin II-Induced Arterial Hypertrophy and PDGF-A. Hypertension 1998; 32:452-458.

40. RUPÉREZ M, LORENZO O, BLANCO-COLIO L, ESTEBAN V, EGIDO J, RUIZ-ORTEGA M, et al. Connective tissue growth factor is a mediator of angiotensin II-induced fibrosis. Circulation 2003; 108, 1499-1505.

41. ZHAO Q, ISHIBASHI M, HIASA K, TAN C, TAKESHITA A, EGASHIRA K, et al. Essential role of vascular endothelial growth factor in angiotensin II-induced vascular inflammation and remodeling. Hypertension 2004; 44:264-270. 
42. TOUYZ R, BERRY C. Recent advances in angiotensin II signaling. Braz J Med Biol Res 2002; 35:1001-1015.

43. RENTZSCH B, TODIRAS M, ILIESCU R, POPOVAE, CAMPOS L, OLIVEIRA ML, et al. Transgenic angiotensin-converting enzyme 2 overexpression in vessels of SHRSP rats reduces blood pressure and improves endothelial function. Hypertension 2008; 52:967-973.

44. SANTOS R, SIMOES E, SILVA A, MARIC C, SILVA D, DE BUHR I, et al. Angiotensin-(1-7) is an endogenous ligand for the G protein-coupled receptor Mas. Proc Natl Acad Sci 2003; 100:8258-8263.

45. LI P, CHAPPELL M, FERRARIO C, BROSNIHAN K. ANGIOTENSIN (1-7) augements bradykinin-induced vasodilation by competing with $\mathrm{ACE}$ and releasing nitric oxide. Hypertension 1997; 29:394-400.

46. YAGIL Y, YAGIL C. Hypothesis: ACE2 modulates blood pressure in the mammalian organism. Hypertension 2003; 41:871-873.

47. ERDÖS E, JACKMAN H, BROVKOVYCH V, TAN F, DEDDISH P. Products of angiotensin I hydrolysis by human cardiac enzymes potentiate bradykinin. J. Moll. Cell. Cardiol 2002; 34:1569-1576.

48. SCHMAIER A. The kallikrein-kinin and the renin-angiotensin systems have a multilayered interaction Am J Physiol Regulatory Integrative Comp Physiol 2003; 285:1-13.

49. GROBE J, MECCA A, LINGIS M, SHENOY V, BOLTON T, MACHADO JM, et al. Prevention of angiotensin II-induced cardiac remodeling by angiotensin-(1-7). Am J Physiol Heart Circ Physiol 2007; 292:H736-H742.

50. IWATAM,COWLING R, GURANTZD, MOOREC,ZHANG S, YUAN JX, et al. Angiotensin-(1-7) binds to specific receptors on cardiac fibroblasts to initiate antifibrotic and antitrophic effects. Am J Physiol Heart Circ Physiol 2005; 289:2356-2363.

51. KRAMKOWSKI K, MOGIELNICKI A, LESZCZYNSKA A, BUCZKO W. Angiotensin-(1-9), the product of angiotensin I conversion in platelets, enhances arterial thrombosis in rats. $\mathrm{J}$ Physiol Pharmacol 2010; 61:317-24.

52. IWAI M. AND HORIUCHI M. Devil and angel in the reninangiotensin system: ACE-angiotensin II-AT1 receptor axis vs ACE2-angiotensin-(1-7)-Mas receptor axis. Hypertension Research 2009; 32:533-536. 